\title{
Pesquisa y tratamiento precoz de los trastornos del lenguaje en niños
}

\author{
Screening and treatment of speech and lenguage problems in childrem.
}

Wallace I y col. 2015;136(2):e448-62.

\section{Objetivos}

Actualizar la evidencia disponible sobre la precisión de los métodos de pesquisa de trastornos del lenguaje y sobre la efectividad de su tratamiento.

\section{Diseño y fuente de datos}

Revisión sistemática para la que fue realizada una búsqueda en MEDLINE, Cochrane Database of Systematic Reviews, Psyclnfo, Cumulative Index to Nursing, clinicalTrials.gov y en listas de referencias. Fueron incluidos estudios que habían comunicado la exactitud diagnóstica de herramientas de pesquisa y ensayos clínicos aleatorizados que habían documentado los riesgos y los beneficios del tratamiento precoz de los pacientes con trastornos del lenguaje.

En forma independiente, dos revisores extrajeron los datos y eva- luaron la calidad los trabajos usando criterios predefinidos.

\section{Resultados Principales}

Los instrumentos (ver tabla 1) de pesquisa administrados a padres para detectar un trastorno del lenguaje presentaron una mediana de sensibilidad de $81 \%$ y de especificidad de $87 \%$; con coeficientes de probabilidad (en inglés LR) positivos y negativos de rangos amplios: LR+ (de 2,4 a 12,4) y LR- (de 0,08 a 0,48).

Los instrumentos administrados por personal entrenado presentaron una mediana de sensibilidad de $74 \%$ y de especificidad de $91 \%$. Algunas herramientas (CDI, ITC, y SKOLD) mostraron una buena consistencia y niveles aceptables de sensibilidad y especificidad (mayores a 70\%) para cada estrato de edad, a diferencia de otras (ASQ, Fluharty, Preschool Speech and Language Screening Test), que tuvieron baja sensibilidad a diferentes edades.

Tabla 1.Características operativas de instrumentos administrados por los padres y por personal entrenado

\begin{tabular}{|c|c|c|c|c|c|c|c|c|c|c|}
\hline $\begin{array}{l}\text { Administrador del } \\
\text { instrumento }\end{array}$ & LR - & $\mathbf{L R}+$ & VPN & VPP & Prevalencia & Especificidad & Sensibilidad & Edad & n & Escala \\
\hline Padres & $\begin{array}{l}0,48 \\
0,23 \\
0,15 \\
\end{array}$ & $\begin{array}{l}2,4 \\
3,9 \\
3,5 \\
\end{array}$ & $\begin{array}{l}92 \\
89 \\
92 \\
\end{array}$ & $\begin{array}{l}32 \\
70 \\
65\end{array}$ & $\begin{array}{l}16 \% \\
38 \% \\
35 \% \\
\end{array}$ & $\begin{array}{l}73 \text { (64 a 82) } \\
79 \text { (69 a 89) } \\
74 \text { (66 a 83) } \\
\end{array}$ & $\begin{array}{l}87(45 \text { a } 88) \\
81(69 \text { a } 94) \\
89(80 \text { a } 97)\end{array}$ & $\begin{array}{c}4,5 \text { años } \\
24 \text { meses } \\
12 \text { a } 17 \text { meses }\end{array}$ & $\begin{array}{l}110 \\
100 \\
151 \\
\end{array}$ & $\begin{array}{c}\text { ASQ } \\
\text { CDI WS } \\
\text { IT }\end{array}$ \\
\hline $\begin{array}{l}\text { Personal } \\
\text { entrenado }\end{array}$ & $\begin{array}{l}0,89 \\
0,53\end{array}$ & $\begin{array}{l}1,8 \\
N R\end{array}$ & $\begin{array}{l}89 \\
15\end{array}$ & $\begin{array}{c}26 \\
100\end{array}$ & $\begin{array}{l}16 \% \\
92 \%\end{array}$ & $\begin{array}{c}70(60 \text { a } 79) \\
100(100 \text { a } 100)\end{array}$ & $\begin{array}{l}56 \text { (33 a 78) } \\
46 \text { (34 a 58) }\end{array}$ & $\begin{array}{c}4,5 \text { años } \\
18 \text { a } 66 \text { meses }\end{array}$ & $\begin{array}{c}110 \\
71\end{array}$ & $\begin{array}{l}\text { Battelle } \\
\text { Denver }\end{array}$ \\
\hline
\end{tabular}

Vale destacar que 13 de los ensayos clínicos documentaron efectividad del tratamiento de niños con trastorno del lenguaje y de la fluencia. Sin embargo la confiabilidad de los resultados es limitada, debido a que la mayoría de estos trabajos incluyeron pocos pacientes, presentaron amplia variabilidad entre estudios -edades, tipo de entrenamiento, personal que realizó los tratamientos, intensidad, estrategia y contenidos de la intervención-, evaluaron diferentes medidas de resultado y reportaron los diferentes resultados en forma inconsistente.

\section{Conclusiones}

Algunos instrumentos mostraron tener buena precisión para la detección de trastornos del lenguaje, con amplios rangos de sensibilidad y especificidad y variaciones en diferentes edades. A partir de esta revisión sistemática los autores no encontraron evidencia de mejor pronóstico en niños tratados precozmente, detectados a partir de estrategias de pesquisa de estas condiciones.

Fuente de finaciamiento: Agency for Healthcare Research and Quality, U.S. Department of Health and Human Services Contract. Los autores no reportaron conflictos de intereses.

\section{Comentario}

El presente estudio revisó la precisión de las herramientas de pesquisa para la detección precoz de trastornos del lenguaje e intentó responder, mediante una revisión sistemática de la bibliografía, si la detección temprana mediante un instrumento de pesquisa resulta en una mejora relevante en comparación con la detección dentro de un control de rutina. No se han publicado hasta el momento trabajos que hayan incluido un número suficiente de pacientes, que hayan controlado adecuadamente sesgos y confundidores y que hayan explicitado el abordaje terapéutico utilizado. Por esta razón resulta aún inapropiado realizar recomendaciones de pesquisa, hasta no que no contemos contrabajos que documenten la efectividad del tratamiento precoz ${ }^{1}$.

\section{Conclusiones del comentador}

Aún no se ha documentado el beneficio de la pesquisa de trastornos del desarrollo en niños -trastornos del espectro autista, trastornos del lenguaje, trastornos específicos del aprendizajerespecto del pronóstico de estos pacientes. Asimismo, la inclusión de un niño en un proceso de evaluación y tratamiento, a partir de resultados de pruebas de pesquisa, puede colocar al niño y a su familia en una situación de estrés, muchas veces innecesaria, y como consecuencia de un resultado falso positivo. La vigilancia de las pautas madurativas en los controles de salud, así como las preocupaciones familiares o del personal escolar ante un posible retraso o trastorno del desarrollo, continúan siendo los elementos de elección para la realización de estudios diagnósticos.

Celeste Puga [ Neuropediatría-Hospital Italiano de Buenos Aires celeste.puga@ hospital.italiano.org.ar ]

Puga C. Pesquisa y el tratamiento precoz de los trastornos del lenguaje en niños. Evid Act Pract Ambul 2017;20(3):Jul-Sep. 75. Comentado de: Wallace I y col. Screening for Speech and Language Delay in Children 5 Years Old and Younger: A Systematic Review. Pediatrics. 2015;136(2):e44862.PMID: 26152671. 